\title{
Linear and Nonlinear analysis of Cardiovascular Time Series during Different Sleep Stages
}

\author{
Lianrong Zheng ${ }^{1,2,3}$, Yifan $\mathrm{Li}^{1,2,3}$, Weifeng Pan ${ }^{1,2,3}$, Kunyang $\mathrm{Li}^{1,2,3}$, \\ guanzheng $\mathrm{Liu}^{1,2,3 *}$
}

${ }^{1}$ School of Engineering, Sun Yat-sen University, Guangzhou 510275, China;

${ }^{2}$ Key Laboratory of Sensing Technology and Biomedical Instrument of Guangdong Province, School of Engineering, Sun Yat-sen University, Guangzhou 510275, China;

${ }^{3}$ Guangdong Provincial Engineering and Technology Centre of Advanced and Portable Medical Device, Guangzhou 510275, China

Keywords: cardiovascular system, heart rate variability (HRV), pulse rate variability (PRV), pulse transit time (PTT), sleep stage.

\begin{abstract}
Sleep is a complex phenomenon involved important modifications throughout life and changes of autonomic cardiovascular control. In present study, we aimed to analyze the influence of sleep stages on cardiovascular activity. Firstly, heart rate variability (HRV), pulse rate variability (PRV) and pulse transit time (PTT) were derived from ECG and PPG signals in 10 heathy volunteers during overnight polysomnography. Then, we extracted linear and nonlinear features of HRV, PRV and PTT. Linear features were consisted of mean intervals, the standard deviation of all normal interval (SDNN) and coefficient of variation (CV), and nonlinear features including approximate entropy (ApEn), sample entropy (SampEn), and fuzzy entropy (FuzzyEn). The results indicated that the SDNN and CV were significantly larger in rapid eye movement (REM) sleep compared to slow wave sleep (SWS) $(\mathrm{p}<0.05)$ in HRV, PRV and PTT. ApEn was significantly larger in REM compared to SWS $(\mathrm{p}<0.05)$ in HRV, PRV. In conclusion, the cardiovascular fluctuations and complexes were decreased when the sleep state progressed from REM to SWS, and the results of linear and nonlinear feathers extracted by PRV were consistent with HRV.
\end{abstract}

\section{Introduction}

Sleep is a complex phenomenon involved important modifications throughout life and changes of autonomic cardiovascular control. HRV is regulated by autonomic nervous system (ANS) and contains abundant information on cardiovascular regulation [1]. PRV provides not only ANS activities but also the information of peripheral circulation [2]. And PTT is dependent on arterial stiffness which is affected by systolic arterial pressure [3]. Therefore, HRV, PRV and PTT were widely applied to assess cardiovascular system.

The influence of sleep stage on HRV has been widely studied. Frequency analysis of HRV demonstrated an increase in HF components and a decrease in LF components in non-rapid eye movement stages, and the opposite changes during rapid eye movement (REM) sleep [4,5]. According to the standard definitions of HRV indices, the linear and nonlinear indices were widely used for analyzing PRV on account of the pulsatile feature of PPG waveform is synchronized with the heart rate [2]. PTT has been reported to be correlated with blood pressure, especially for the systolic blood pressure (SBP), and influenced by sleep stages [3,6]. In contrast, the details of 
dynamics in HRV, PRV and PTT have not been investigated. In term of linear and nonlinear analysis, a few studies compared the differences of indices fluctuations among cardiovascular time series during sleep.

\section{Materials and methods}

\subsection{Data collection}

In our study, we selected 10 healthy volunteers (43.5 \pm 11.55 years old) who underwent full nocturnal polysomnographic (PSG) sleep recordings. The PSG recorded (Alice 5 PSG, Philips, Inc) six electroencephalogram (EEG) channel, left and right electrooculogram (EOG), submental electromyogram (EMG), lead I and II ECG $(500 \mathrm{~Hz})$, PPG $(100 \mathrm{~Hz})$ and so on. According to the standardized EEG, EOG, and EMG criteria of Rechtschaffen and Kales (R\&K rules), for each subject the sleep stages (stage REM, N1, N2 and N3) were scored visually in 30-s epochs by an experienced sleep medicine specialist.

\subsection{Data analysis}

Based on Pan-Tompkins algorithm [7], HRV was obtained by measuring the intervals between the successive R peaks of ECG. PRV was computed as the intervals between consecutive peaks in the PPG signal. PTT usually calculated as the time interval between the R-peak of ECG and the peak of the PPG detected within the same cardiac cycle. In this study, N1 and N2 are called as light sleep stage (LS), and stages, and N3 is called as slow wave sleep (SWS). All time series were performed at five-minute segment without sleep events. In order to assess the cardiovascular activity during sleep, the specific features of linear analysis and nonlinear analysis were applied to characterize autonomic cardiovascular control. Linear analysis: Time-domain measurements including the mean interval, SDNN, CV; Information theory measurements including ApEn, SamEn and FuzzyEn [8]. Entropy measures complexity (or regularity) of a random signal. A high value of entropy reflects a high degree of complexity.

\section{Results}

As show in Table1, subjects displayed a normal sleep architecture.

Table 1. Polygraphic macrostructural parameters

\begin{tabular}{lcc}
\hline & mean & standard deviation \\
\hline sleep efficiency $(\%)$ & 0.85 & 0.08 \\
REM (\%) & 17.46 & 5.12 \\
N1 (\%) & 15.77 & 4.72 \\
N2 (\%) & 45.68 & 6.69 \\
N3 (\%) & 11.89 & 5.32 \\
AHI & 4.81 & 5.13 \\
\hline
\end{tabular}

Table 2 summarizes the results of linear and nonlinear HRV analysis. As the result demonstrated in linear analysis, the SDNN and CVh showed monotonic decrease with the sleep deeper. The SDNN and CVh were significantly larger in REM compared to SWS $(\mathrm{p}<0.05)$. However, as compared to CVh ( $\mathrm{p}=0.005)$, the significance level P-value of changed was obvious smaller than that of SDNN ( $\mathrm{p}=0.000)$. In addition, the CVh was significantly larger in REM compared to LS $(\mathrm{p}<0.05)$. Nonlinear analysis demonstrated there was significantly larger ApEn in 
REM as compared to LS and SWS. And the sample entropy of LS was larger than this of SWS. Table 2 The analysis of heart rate variability in different sleep stages (mean \pm SD)

\begin{tabular}{lllll}
\hline & REM & LS & SWS & P value \\
\hline Linear analysis & & & & \\
RRm(ms) & $950 \pm 97$ & $989 \pm 114$ & $1010 \pm 99$ & 0.182 \\
SDNN(ms) & $60 \pm 27$ & $47 \pm 18$ & $38 \pm 13^{*}$ & $\mathbf{0 . 0 0 5}$ \\
CVh(\%) & $0.062 \pm 0.023 \#$ & $0.047 \pm 0.016$ & $0.038 \pm 0.013^{*}$ & $\mathbf{0 . 0 0 0}$ \\
Nonlinear analysis & & & & \\
ApEn(n.u) & $0.030 \pm 0.0068$ & $0.023 \pm 0.010^{*}$ & $0.019 \pm 0.011^{*}$ & $\mathbf{0 . 0 0 2}$ \\
SampEn(n.u) & $1.191 \pm 0.249$ & $1.334 \pm 0.100$ & $1.243 \pm 0.097 \#$ & $\mathbf{0 . 0 2 7}$ \\
FuzzyEn(n.u) & $0.183 \pm 0.053$ & $0.206 \pm 0.075$ & $0.202 \pm 0.061$ & 0.491 \\
\hline
\end{tabular}

* Significantly different from REM ( $\mathrm{p}<0.05)$; \# Significantly different from LS ( $<<0.05)$; n.u: no units

Table 3 summarizes the results of linear and nonlinear PRV analysis. As the result demonstrated in linear analysis, the SDPP and CVp showed monotonic decrease with the sleep deeper. The SDPP and CVp had significantly larger in REM compared to LS and SWS $(\mathrm{p}<0.05)$. Nonlinear analysis demonstrated there was significantly larger ApEn in REM as compared to SWS. However, there was no the significant difference in SampEn and FuzzyEn among in sleep stages.

Table 3 The analysis of pulse rate variability in different sleep stages (mean \pm SD)

\begin{tabular}{lllll}
\hline & REM & LS & SWS & P value \\
\hline Linear analysis & & & & \\
PPm(ms) & $949 \pm 97$ & $989 \pm 114$ & $1011 \pm 98$ & 0.175 \\
SDPP(ms) & $70 \pm 30$ & $50 \pm 17^{*}$ & $39 \pm 15^{*}$ & $\mathbf{0 . 0 0}$ \\
CVp(\%) & $0.073 \pm 0.027$ & $0.050 \pm 0.016^{*}$ & $0.039 \pm 0.016^{*}$ & $\mathbf{0 . 0 0}$ \\
Nonlinear analysis & & & & \\
ApEn(n.u) & $0.031 \pm 0.018$ & $0.021 \pm 0.012$ & $0.019 \pm 0.009^{*}$ & $\mathbf{0 . 0 2 1}$ \\
SampEn(n.u) & $1.194 \pm 0.118$ & $1.281 \pm 0.158$ & $1.240 \pm 0.226$ & 0.297 \\
FuzzyEn(n.u) & $0.205 \pm 0.054$ & $0.215 \pm 0.080$ & $0.209 \pm 0.056$ & 0.885 \\
\hline
\end{tabular}

PPm: mean PTT interval; SDPP: standard deviation of PP intervals; * Significantly different from REM $(\mathrm{p}<0.05)$; \# Significantly different from LS $(\mathrm{p}<0.05)$

Table 4 The analysis of pulse transit time variability in different sleep stages (mean $\pm \mathrm{SD}$ )

\begin{tabular}{lllll}
\hline & REM & LS & SWS & P value \\
\hline Linear analysis & & & & \\
PTTm(ms) & $3.086 \pm 0.204$ & $3.046 \pm 0.189$ & $3.124 \pm 0.204$ & 0.602 \\
SD(ms) & $0.086 \pm 0.051$ & $0.056 \pm 0.034$ & $0.052 \pm 0.033^{*}$ & $\mathbf{0 . 0 1 8}$ \\
CV(\%) & $0.027 \pm 0.0164$ & $0.018 \pm 0.010^{*}$ & $0.016 \pm 0.008^{*}$ & $\mathbf{0 . 0 1}$ \\
Nonlinear analysis & & & & \\
ApEn(n.u) & $0.048 \pm 0.043$ & $0.025 \pm 0.013$ & $0.023 \pm 0.020$ & $\mathbf{0 . 0 1 7}$ \\
SampEn(n.u) & $1.091 \pm 0.312$ & $1.218 \pm 0.206$ & $1.182 \pm 0.225$ & 0.268 \\
FuzzyEn(n.u) & $0.195 \pm 0.065$ & $0.196 \pm 0.048$ & $0.215 \pm 0.061$ & 0.502 \\
\hline
\end{tabular}

PTTm: mean PTT; SD: standard deviation of PTT; * Significantly different from REM ( $<<0.05)$;

\# Significantly different from LS ( $\mathrm{p}<0.05)$

Table 4 summarizes the results of linear and nonlinear PTTV analysis. As the result 
demonstrated, the SDNN and CV showed monotonic decrease with the sleep deeper. The SDNN and CV were significantly larger in REM compared SWS $(\mathrm{p}<0.05)$. In addition, the CV was significantly larger in REM compared LS $(\mathrm{p}<0.05)$. However, there was no the significant difference in ApEn, SampEn and FuzzyEn among in sleep stages.

\section{Discussion and conclusions}

In this study, we estimated the linear and nonlinear features of HRV, PRV and PTT derive from ECG and PPG signals during overnight polysomnography. The cardiovascular fluctuations (SD and CV) was decrease when the sleep state progressed from REM to SWS. And the degree of complexes of cardiovascular activity was decrease when the sleep state progressed from REM to SWS. Therefore, autonomic cardiovascular control was successfully confirmed by above-mentioned linear and nonlinear feathers. Respiratory has a key role in cardiovascular system [9-11]. In the future, based on multivariate dynamic analysis, we will have a further research in the interrelationship in cardiorespiratory.

\section{Acknowledgments}

The authors would like to acknowledgment the support from the National Natural Science Foundation of China (61401521) and Natural Science Foundation of Guangdong province (2014A030310163).

\section{References}

[1] G.Z. Liu, L. Wang, Q. Wang, et al, A new approach to detect congestive heart failure using short-term heart rate variability measures, Plos One. 9 (2014) e93399.

[2] P. Dehkordi, A. Garde, W. Karlen, et al. Pulse rate variability in children with disordered breathing during different sleep stages, Computing in Cardiology Conference (CinC). (2013) 1015-1018.

[3] L.C. Nisbet, S.R. Yiallourou, S.N. Biggs, et al, Preschool children with obstructive sleep apnea: the beginnings of elevated blood pressure sleep, Sleep. 36 (2013) 1219-1226.

[4] M. Tsunoda, T. Endo, S. Hashimoto, et al, Effects of light and sleep stages on heart rate variability in humans, Psychiatry \& Clinical Neurosciences. 55 (2001) 285-286.

[5] P. Busek, J. Vanková, J. Opavský, et al, Spectral analysis of the heart rate variability in sleep, Physiol. Res. 54(2005) 369-76.

[6] Vlahandonis, S.N. Biggs, G.M. Nixon, et al, Pulse transit time as a surrogate measure of changes in systolic arterial pressure in children during sleep, J. Sleep. Re. 23 (2014) 406-13.

[7] W.H. Chen, L.R. Zheng, K.Y. Li, et al, A novel and effective method for congestive heart failure detection and quantification using dynamic heart rate variability measurement, Plos One. 11 (2016) e0165304.

[8] J. Pan, W.J. Tompkins, A real-time QRS detection algorithm, IEEE Transactions on Biomedical Engineering. 32 (1985) 230-236.

[9] G.Z. Liu, G.M. Zhou, W.H. Chen, et al. A principal component analysis based data fusion method for estimation of respiratory volume, IEEE Sensors Journal. 15 (2015) 4355-4364.

[10]G.Z. Liu, B.Y. Huang, L. Wang, A wearable respiratory biofeedback system based on generalized body sensor network, Telemedicine Journal and E-health. 17 (2011) 348-357. 
[11]S.J. Redmond, C. Heneghan, Cardiorespiratory-based sleep staging in subjects with obstructive sleep apnea, IEEE Transactions on Biomedical Engineering. 53 (2006) 485-496. 